\title{
Dynamische Erscheinungen an Grenzflächen zwischen fluiden Phasen
}

Unter dem vorgenannten Thema fand vom 30.3.-1.4.81 die Diskussions-Tagung der Deutschen Bunsengesellschaft statt. Die Vorträge sind im Band 85 (1981), Heft 10, S. 826-919 wiedergegeben. Sie dürften die Arbeitsgebiete vieler CPS-Leser direkt berühren. Auf den Inhalt sei in kurzen Stichworten hingewiesen.

„Wechselwirkungskräfte in flüssigen Dünnschichten" von J. Lyklema, S. 826-833 (Ủbersicht über die in dünnen flüssigen Filmen kolloidaler Dimensionen wirkenden Kräfte; von der Waals-Kräfte; elektrostatische Wechselwirkung; Gleichgewichtsdicken ohne sterische Effekte; sterische Wechselwirkungsbeiträge; Anwendung unter dynamischen Bedingungen; Einfluß der Gegenwart von Tensiden und Polymeren). „Entropieerzeugende Prozesse an Phasengrenzflächen" von M. J. Hampe, S. 834-842 (thermodynamische Theorie zur Behandlung der Transporterscheinungen in mehrphasigen und mehrkomponentigen Systemen; Beschreibung der Struktur einer dreidimensionalen Grenzfläche mittels der irreversiblen Thermodynamik; ungleiche Entropieeigenschaften ein- und mehrphasiger Systeme; Marangoni-Effekt). „Fluide Grenzflächen und Lamellen in der Technik" von H. Nassenstein, S. 842-847 (Ubersicht unter Anführung von 14 verfahrenstechnischen Gebieten und deren Grenzflächenprobleme, behandelt werden die Grenzflächen flüssig/gasförmig, flüssig/flüssig, flüssig/fest; Herstellung dünner Uberzüge auf Festkörpern). „Ellipsometrie - Eine Methode zur Charakterisierung dünner Filme von W. Haller, S. 847-851 (Grundlagen: Polarisation des Lichtes, ellipsometrische Meßanordnung, Reflexion von polarisiertem Licht, manuelle und automatische Ellipsometer; Anwendung). „Koaleszenz in dichtgepackten Gas/flüssig- und flüssig/flüssig-Dispersionen" von S. Hartland, S. 851-863 (Beispiete für Koaleszenz, Beschreibung typischer Phänomene; Schäume im stationären Zustand, Zerfall eines Schaumes; flüssig/flüssig-Dispersionen im stationären Zustand, zerfallende und wachsende Dispersionen; 129 Zitate). „Dynamic Processes in Water-in-Oil-Microemulsions" von P.D. I. Fletscher et al, S. 863-867 (Untersuchung mittels Kleinwinkel-Neutronenstreuung und Fluoreszenz, Kinetik des Uberganges einer solubilisierten Komponente in Wasser-Ol-Mikroemulsionen; Bedetung solcher Untersuchungen). "Emulsions in anhanced Oil Recovery" von F. T. Hesselink, S. 867-868 (tertiäre Erdölförderung, Emulsionen, Oberflächenerscheinungen). „Investigation of Electron Transfer Reactions of Metal-Porphyrin Complexes in Micellar Solutions" von V. Eck et al., S. 869-876 [als Elektronendonatoren dienten die Ag-Komplexe von 3 Porphyrinen und als Akzeptoren $\mathrm{IrCl}_{6}^{2-}$ oder $\mathrm{Os}$ (dipy) $3_{3}^{3+}$; Bestimmung der Geschwindigkeitskonstanten in wäßriger Lösung, Einfluß von Micellen aus Tensiden und der Ionenstärke der Lösung; zahlreiche Einzelergebnisse]. "The Infiuence of Counter-Ion-Concentration on the Aggregation Behaviour of Viscoelastic Detergents" von H. Hoffmann et al., S. 877-882 [Einfluß von Na-Salicylat (I) auf die viscoelastischen Eigenschaften der Lösungen von Cetylpyridiniumsalicylat (II); statische und dynamische Lichtstreuung, elektrische Doppelbrechung, rheologische Messungen; Stäbchenlänge von II steigt mit wachsender I-Konzentration bis $200 \mathrm{~nm}$ an, schließlich Knäuelbildung von II]. ,Elektronenmikroskopische Untersuchungen von Micellen" von L. Bachmann et al., S. 883-887 (wäßrige Micellen von Eicosyl- und Docosylsulfat, Docosylpyridiniumbromid, Hexadecyl- $N$-glycin, Sucrosemonomyristat und -palmitat; Micellengewicht, Aggregationszahl $N$, kritische Micellkonzentration cmc, Micelldurchmesser $D ; N$ unabhängig von Konzentration bis $20 \mathrm{cmc}, D$ meist $>8 \mathrm{~nm}$ ). "Neutron Scattering from Concentrated Micellar Solutions" von J. B. Hayter, S. 887-891 (wäßrige Lösungen von $\mathrm{Na}$-Dodecylsulfat $+\mathrm{NaCl}$; Strukturparameter; dynamische Neutronen-Spinecho-Spektroskopie). "The Surfactant-Block Structure of Micelles, Synthesis of the Droplet and of the Bilayer Concept" von P. Fromherz, S. 891-899 (Micellen aus Na-Dodecylsulfat bzw. Cetyltrimethylammoniumchlorid, Stöchiometrie, Struktur, energetische Verhältnisse, radiale und nichtradiale Packung; das Oberflächen-Blockmodell, Aufbau, Stöchiometrie, Wachstum, Solubilisation; andere Bildungen). „Kinetik der Stoffübertragung “ von W. Nitsch, S. 899-900 (Grenzflächenreaktionen, Oberflächenerscheinungen, Reaktionskinetik, Transporterscheinungen). „Einfluß von Tensiden auf Strömung und Stoffübergang in einer flüssig/flüssig-Kanalströmung" von R. Ollenik et al., S. 900-904 (Transport von Aceton durch die $\mathrm{H}_{2} \mathrm{O} / \mathrm{CCl}_{4}$ Grenzfläche in Gegenwart von Na-Dodecylsulfat; für $c<\mathrm{cmc}$ ist der Acetontransport unterdrückt, steigt aber mit steigendem $c$ ab cme stark an; Bestimmung des Grenzflächendrucks). „Interfacial

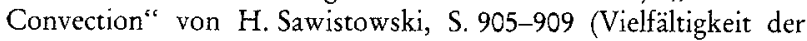
Phänomene, unterteilt gemäß Marangoni-Instabilität, MarangoniStörungen, Stabilität dünner Filme; praktische Anwendungen). „Conductive and Convective Heat Transfer below Evaporating Liquid Surfaces" von D. Schreiber et al., S. 909-914 (Meßmethode, Messungen an verdampfendem Wasser, kritische Rayleigh-Zahl für den Ubergang vom laminaren zum turbulenten Fluß; zahlreiche Einzelergebnisse). „Mass and Charge Transfer across the Organic/ Aqueous Phase-Boundary in Electrolyis Processes using TwoPhase-Emulsion Mixed Electrolytes" von H. Feess et al., S. 914-919 (Hinsichtlich des Stoff- und Ladungsdurchtritts durch die Grenzfläche bei der Elektrolyse von zweiphasigen Emulsionen werden 4 verschiedene Typen unterschieden, die unter Verwendung mehrerer Systeme untersucht wurden; die technische Bedeutung der Effekte).

Anschrift des Verfassers:

Prof. Dr. O. Fuchs

Lessingsstr. 24

6238 Hofheim/Taunus 